\title{
Insatisfação corporal e qualidade de vida durante a menarca e sua relação com a renda familiar e o índice de massa corporal: Um estudo longitudinal
}

\author{
Body dissatisfaction and life quality during the menarche and its relation \\ to the family income and the body mass index: A longitudinal study
}

\author{
Mara Lucia Blanc Santos ${ }^{1 *}$, Jefferson Silva Novaes ${ }^{2}$, Lilian Alves da Costa Monteiro ${ }^{1}$, \\ Hélder Miguel Fernandes ${ }^{3}$
}

ARTIGO ORIGINAL | ORIGINAL ARTICLE

\begin{abstract}
Este estudo analisou a associação entre a renda familiar e níveis de índice de massa corporal com a imagem corporal e qualidade de vida ao longo da menarca; e a variação da relação entre a renda familiar e o IMC com a imagem corporal e a qualidade de vida em distintos momentos da menarca (momentos 1,2 e 3). O estudo caracterizou-se por um delineamento longitudinal com adolescentes de 10 a 13 anos, IMC de $18.98 \mathrm{~kg} / \mathrm{m}^{2}$. Os dados foram coletados pelo Body Shape Questionnaire e Autoquestionnaire Qualité de Vie Enfant Imagé no momento 1 no ano de 2010, no momento 2 no ano de 2011 e no momento 3 no ano de 2012. Os principais resultados indicaram: que o IMC se relacionou positivamente com o escore total de insatisfação corporal no momento 1,2 e 3; a análise nos níveis de renda familiar e IMC indicaram melhoras positivas ao longo da menarca; e o escore de qualidade de vida aumentou do momento 1 para o 2, tendo reduzido do momento 2 para o 3 . Concluiu-se que as adolescentes revelaram uma menor insatisfação com a imagem corporal com o passar do tempo, evidenciada por um IMC normal; e ao longo do tempo a baixa insatisfação da imagem corporal revelouse pela associação positiva da renda familiar e do IMC. Já a qualidade de vida apresentou-se melhor entre o momento 1 e 2 da menarca, mas entre o momento 2 e 3 ocorreu uma diminuição da qualidade de vida das adolescentes.

Palavras-chaves: menarca, imagem corporal, peso corporal, qualidade de vida
\end{abstract}

ABSTRACT

This study analysed the association between family income and body mass index levels with body image and quality of life along the menarche; and the variation of the relation between family income and BMI with body image and life quality at different moments of the menarche (moments 1, 2 and 3). The study was characterized by a longitudinal delimitation involving teenagers aged 10 to 13, BMI 18.98 $\mathrm{kg} / \mathrm{m}^{2}$. The data was collected through the Body Shape Questionnaire and Autoquestionnaire Qualité de Vie Enfant Image at moment 1 in 2010, at moment 2 in 2011 and at moment 3 in 2012. The main results showed BMI positively related to the total score of body dissatisfaction in moments 1, 2 and 3; analysis on family income levels and BMI showed positive improvements along the menarche; and life quality score raised from moment 1 to 2 , having decreased from moment 2 to 3 . The conclusion was that adolescents showed lower dissatisfaction with body image as time went by, enhanced by a normal BMI; and as time went by the low body image dissatisfaction came out by the positive association between family income and BMI. Quality of life was better between moments 1 and 2 of the menarche, but there was a decrease in the life quality of the adolescents.

Keywords: menarche, body image, body weight, life quality

\footnotetext{
Artigo recebido a 23.03.2014; Aceite a 12.11.2014

${ }^{1}$ Departamento de Ciências do Desporto, Exercício e Saúde, Universidade de Trás-os-Montes e Alto Douro, Vila Real, Portugal

${ }^{2}$ Universidade Federal do Rio de Janeiro, Rio de Janeiro, Brasil

${ }^{3}$ Centro de Investigação em Desporto, Saúde e Desenvolvimento Humano, CIDESD, Portugal

* Autor correspondente: Rua Rio Araguaia, 1645. Bairro: Fazendinha. Macapá-Amapá-Brasil.

E-mail: mara.blanc@hotmail.com
} 


\section{INTRODUÇÃO}

A menarca, denominada primeira menstruação, é um marco na vida reprodutiva da mulher e considerada um fator biológico importante na adolescência e na fase adulta (Sloboda, Hart, Doherty, Pennell, \& Hickey, 2007). Sendo um indicador de maturação biológica da mulher, a menarca ainda é utilizada para mostrar mudanças ambientais, socioeconômicas, culturais e psicológicas ocorrentes nas adolescentes. Sabe-se que 95\% das adolescentes apresentam a menarca entre os 11 e 15 anos (Roman, Ribeiro, GuerraJúnior, \& Barros-Filho, 2009) e que está variabilidade está provavelmente relacionada a fatores climáticos, localização geográfica, estado nutricional, nível socioeconômico, entre outros (Al-Sahab, Ardern, Hamadeh, \& Tamim, 2010; Scherer, Martins, Pelegrini, Matheus, \& Petroski, 2010).

Roman, Ribeiro, Guerra-Júnior, e BarrosFilho (2009) ao relacionarem a questão socioeconômica com a menarca, evidenciaram que adolescentes de maior nível socioeconômico apresentaram a idade da menarca antes das adolescentes de médio e baixo nível socioeconômico, sustentando que a relação da tendência secular com a idade da menarca pode ser atribuída às melhores condições sociais e de renda nos últimos anos (Hosny et al., 2005). E ainda, estudos com meninas de nível socioeconômico inferior também indicaram que a menarca pode ser retardada por fatores socioambientais e nutricionais durante a infância e a adolescência (Orden, Vericat, \& Apezteguía, 2011). A antecipação da menarca pode ainda estar associada com a melhora do nível socioeconômico e maior prevalência de sobrepeso e obesidade nessa fase da vida (Hernández, Unanue, Gaete, Cassorla, \& Codner, 2007).

Investigações relacionando a menarca com o índice de massa corporal (IMC) revelaram que adolescentes com maior IMC menstruaram precocemente, sendo que as adolescentes com IMC menor apresentaram uma menstruação tardia. Desse modo, podemos evidenciar uma interrelação de índices elevados de IMC com a antecipação da menarca (Castilho, Pinheiro, Bento, Barros-Filho, \& Cocetti, 2012; Hernández et al., 2007). Já os estudos de Santos, Monteiro, Silva, Sousa, e Novaes (2009) relataram que adolescentes com IMC baixo apresentaram uma insatisfação corporal e as adolescentes com IMC normal e sobrepeso não revelaram níveis de insatisfação corporal na pós menarca. Entretanto, um estudo em que compararam os níveis de satisfação de imagem corporal de adolescentes antes e depois da menarca, evidenciaram que a maioria das adolescentes com um IMC normal, apresentou uma menor insatisfação com a imagem corporal (Santos et al., 2012). Assim, supõe-se que o nível de IMC para maior ou menor poderá de algum modo influenciar a imagem corporal de adolescentes.

Um estudo sobre a imagem corporal associada com a maturação sexual revelou que a presença da menarca e sua ocorrência em idade precoce fazem com que as adolescentes apresentem o desejo de reduzir o peso corporal (Scherer et al., 2010). Assim, parece que a insatisfação da imagem corporal acarreta sentimentos e pensamentos negativos quanto à aparência e forma do corpo, influenciando possivelmente o bem-estar emocional e a qualidade de vida das adolescentes (Del Ciampo \& Del Ciampo, 2010).

Finalmente, revendo a literatura observa-se que aspectos positivos e negativos referentes à ocorrência da menarca são pouco esclarecedores, revelando a escassez de pesquisas longitudinais que apresentem a influência da renda familiar, índice de massa corporal, imagem corporal e qualidade de vida de adolescentes em diferentes momentos da menarca. Portanto, formulam-se as seguintes questões: Será que existe uma associação entre a renda familiar e os níveis de IMC com a imagem corporal e qualidade de vida em diferentes momentos da menarca? Será que a relação entre a renda familiar, o IMC, a 
imagem corporal e a qualidade de vida variam ao longo do tempo?

Desse modo, este estudo longitudinal pretendeu: a) analisar a associação entre a renda familiar e níveis de índice de massa corporal com a imagem corporal e qualidade de vida ao longo da menarca; e analisar a variação da relação entre a renda familiar e o IMC com a imagem corporal e a qualidade de vida em distintos momentos da menarca (momentos 1, 2 e 3$)$.

\section{MÉTODO}

\section{Amostra}

O processo de amostragem foi do tipo não probabilístico (por conveniência) e o estudo caracterizou-se pelo delineamento longitudinal envolvendo adolescentes do sexo feminino de 10 a 13 anos $(M=11.03 ; D P=0.78)$ com um IMC de $18.98 \mathrm{~kg} / \mathrm{m}^{2}(D P=3.17)$. Foi coletado um total de 388 adolescentes no primeiro momento, entretanto ao longo do estudo a amostra final permaneceu composta por 136 adolescentes que não haviam menstruado. Desse modo o restante $(n=252)$ foi excluído da amostra definitiva, por não cumprirem este critério de inclusão. Os critérios de inclusão foram: a menstruação não ter acontecido até o primeiro momento da coleta de dados, a voluntariedade das participantes e a assinatura do Termo de Consentimento Livre e Esclarecido preenchido corretamente por seus responsáveis. Os critérios para exclusão foram: se ausentar da pesquisa durante os momentos determinados pelo estudo ou não responder os questionários definidos para coleta de dados.

Foi aplicada uma anamnese para obter informações sobre os dados como data de nascimento, idade, cidade/estado, nome da escola, série escolar das adolescentes e a presença (sim) ou ausência (não) da menarca em cada momento da pesquisa; e ainda foram registradas informações sobre $\mathrm{o}$ aspecto socioeconômico, mensurado através da renda familiar que variou de um salário mínimo ou/a acima de cinco salários mínimos. No que diz respeito ao agregado familiar, as adolescentes deveriam marcar a resposta que estava relacionada com o tipo de família em que vivia, como: viver com os pais e outros membros (irmãos, tios, avós), viver só com o pai e mãe ou indicavam somente viver com a mãe ou com o pai. Quanto à cor/etnia deveria marcar ter a cor da pele parda, ter a pele morena/preta, ter a pele branca ou ser da raça/etnia indígena.

\section{Instrumentos}

O peso corporal foi mensurado por uma balança com resolução de $100 \mathrm{~g}$ e a estatura por um estadiômetro com escala milimétrica, para cálculos do IMC=peso/estatura ${ }^{2}$. A determinação dos níveis de IMC (normal e sobrepeso) foi definida a partir do cálculo do IMC, segundo as recomendações das referências de Cole, Bellizzi, Flegal, e Dietz (2000) e Conde e Monteiro (2006), adotandose os pontos de corte ajustada pelo sexo, infância e adolescência.

O Body Shape Questionnaire (BSQ), originado por Cooper, Taylor, Cooper, e Fairburn (1987) e validado por Conti, Cordás, e Latorre (2009) para adolescentes, avaliou a insatisfação com a imagem corporal. Este questionário é autoaplicável do tipo escala Likert, com 34 perguntas. As respostas variam de 1 (nunca) a 6 (sempre), sendo a soma das pontuações de cada fator o escore final da escala. Neste estudo, os valores de consistência interna do escore total ficaram entre $0.93 \mathrm{e}$ 0.95. Quanto aos fatores desse instrumento nos três momentos de avaliação, obtiveram-se os seguintes alfas de Cronbach: Fator 1 autopercepção da forma/aparência corporal (> 0.91); Fator 2 - percepção comparativa da imagem corporal (>0.68); Fator 3 - atitudes em relação à alteração com a imagem corporal ( $>0.65)$ e o Fator 4 - alterações severas na percepção da imagem corporal (entre 0.18 e 0.40), sendo o último fator excluído da análise seguinte, pois não foi significativo na amostra.

O Autoquestionnaire Qualité de Vie Enfant Imagé (AUQEI) desenvolvido por Manificat e Dazord (1997); adaptado e validado por 
Assumpção, Kuczynski, Sprovieri, e Aranha (2000) foi utilizado para avaliar a qualidade de vida. Para obtenção de um perfil da satisfação de crianças e adolescentes foram realizadas 26 questões com quatro alternativas de respostas, diante de diferentes situações: autonomia, lazer, função e família. A escala de respostas varia de 0 a 3 respectivamente, e quanto maior os escores significam melhor qualidade de vida. Nesta investigação os fatores de consistência interna situaram-se entre 0.74 e 0.85 para o escore total desse instrumento nos vários momentos da avaliação.

\section{Procedimentos}

O processo da escolha das escolas deu-se por conveniência, foram selecionadas duas escolas públicas em diferentes zonas da cidade de Macapá-Amapá-Brasil. A coleta de dados das variáveis ocorreu, ao longo de dois anos e meio, em três diferentes momentos da menarca:

momento 1 - pré-menarca (março de 2010), fizeram parte da amostra as adolescentes em que não havia ocorrido a primeira menstruação e que atenderam os critérios de inclusão do estudo;

momento 2 - pós-menarca (outubro de 2011), quando já ocorreu a menarca;

momento 3 - um ano após a coleta pósmenarca (outubro de 2012), foi o momento de acompanhamento (follow-up).
O estudo obteve aprovação do Comitê de Ética e Pesquisa Envolvendo Seres Humanos da Universidade Estadual do Pará (UEPA), com o protocolo n. ${ }^{\circ}$ 085.0.412.000-11, de acordo com a Resolução nº196/1996.

\section{Análise estatística}

Procedeu-se a estatística descritiva para a média, desvio padrão e percentagens, coeficientes de assimetria e curtose computados para efeitos de análise de normalidade univariada. Os coeficientes de normalidade univariada de todas as variáveis, em todos os momentos de avaliação, variaram entre -1 e +1 , indicando uma distribuição do tipo normal. Utilizou-se o alfa de Cronbach para verificar a confiabilidade das escalas. A associação entre variáveis nos três momentos da menarca foi determinada pelo coeficiente de $r$ de Pearson. Para estimativa da estatística inferencial foi utilizada a ANOVA one way para análise comparativa, a ANOVA de medidas repetidas e o cálculo do effect size para análise longitudinal e o teste post hoc de Bonferroni para identificar possíveis diferenças, com nível de significância $p<0.05$, sendo realizados pelo programa SPSS 16.0.

\section{RESULTADOS}

Os resultados sobre $\mathrm{o}$ aspecto socioeconômico agregado familiar e cor/etnia das adolescentes, são apresentados na tabela 1 .

Tabela 1

Resultados de renda familiar, agregado familiar e cor/etnia.

\begin{tabular}{|c|c|c|}
\hline Renda familiar & $\mathrm{N}^{\circ}$ de adolescentes & Percentual \\
\hline 1 salário mínimo & 37 & $27.2 \%$ \\
\hline 2 salários mínimos & 39 & $28.7 \%$ \\
\hline 3 salários mínimos & 23 & $16.9 \%$ \\
\hline 4 salários mínimos & 13 & $9.6 \%$ \\
\hline 5 salários mínimos & 24 & $17.6 \%$ \\
\hline \multicolumn{3}{|l|}{ Agregado familiar } \\
\hline Viviam com os pais e outros membros da família & 103 & $75,7 \%$ \\
\hline Viviam com o pai e a mãe & 19 & $14.0 \%$ \\
\hline Viviam somente com o pai ou com a mãe & 14 & $10.3 \%$ \\
\hline \multicolumn{3}{|l|}{ Cor/etnia } \\
\hline Pele parda & 76 & $58.1 \%$ \\
\hline Pele morena/preta & 37 & $25.0 \%$ \\
\hline Pele branca & 21 & $15.4 \%$ \\
\hline Raça/etnia indígena & 2 & $1.5 \%$ \\
\hline
\end{tabular}


A tabela 2 apresenta a análise da percentagem de adolescentes segundo os níveis de IMC, com os seguintes resultados: momento 1 - peso normal (59.6\%) e sobrepeso (40.4\%); momento 2 - peso normal (64.0\%) e sobrepeso (36.0\%); e momento 3 peso normal (70.6\%) e sobrepeso (29.4\%).
Observou-se que o percentual das adolescentes insatisfeitas com a imagem corporal (de acordo com o ponto de corte 111, proposto Cooper et al., 1987) diminuiu ao longo das três avaliações: $\quad 9.6 \%$; $\quad 8.1 \%$ e $\quad 5.9 \%$, respectivamente.

Tabela 2

Resultados do percentual de adolescentes segundo o nível de IMC e insatisfação com a imagem corporal.

\begin{tabular}{cccc}
\hline Momentos da menarca & \multicolumn{2}{c}{ IMC } & IIC \\
\cline { 2 - 3 } & Normal & Sobrepeso & \\
\hline Momento 1 & $59.6 \%$ & $40.4 \%$ & $9.6 \%$ \\
Momento 2 & $64.0 \%$ & $36.0 \%$ & $8.1 \%$ \\
Momento 3 & $70.6 \%$ & $29.4 \%$ & $5.9 \%$ \\
\hline
\end{tabular}

IMC-índice de massa corporal; IIC-insatisfação da imagem corporal

Posteriormente, procedeu-se a análise de correlação entre renda familiar, IMC, insatisfação da imagem corporal e qualidade de vida em momentos distintos da menarca que estão apresentados na tabela 3. No momento 1 - na pré-menarca, a renda familiar não se associou significativamente com as dimensões da insatisfação corporal e qualidade de vida. $\mathrm{O}$ IMC relacionou-se positivamente com o escore total de insatisfação corporal $(r=0.36 ; p<$ 0.001) e suas dimensões específicas: autopercepção da forma/aparência corporal $(r$ $=0.39 ; p<0.001$ ), percepção comparativa da imagem corporal $(r=0.24 ; p=0.005) \mathrm{e}$ atitudes em relação à alteração da imagem corporal $(r=0.20 ; p=0.018)$. A insatisfação corporal não se relacionou significativamente com a qualidade de vida $(p=0.816)$.

No momento 2 - na pós-menarca, a renda familiar não se associou significativamente com a insatisfação corporal e qualidade de vida. O IMC correlacionou-se positivamente com o escore total de insatisfação corporal $(r=0.44$; $p<0.001)$ e seus fatores específicos: autopercepção da forma/aparência corporal $(r$ $=0.42 ; p<0.001)$, percepção comparativa da imagem corporal $(r=0.26 ; p<0.01)$ e atitudes em relação à alteração com a imagem corporal $(r=0.37 ; p<0.001)$. Não se observou relação significativa entre a insatisfação corporal e qualidade de vida ( $p=$ 0.240).

Os resultados recolhidos no momento 3 um ano após a coleta pós-menarca, foram consistentes com a avaliação anterior em que a renda familiar não influenciou a insatisfação corporal e a qualidade de vida, enquanto o IMC se relacionou positivamente com o escore total da insatisfação corporal $(r=0.44 ; p<0.001)$ e seus domínios: autopercepção da forma/aparência corporal $(r=0.44 ; p<$ 0.001), percepção comparativa da imagem corporal $(r=0.32 ; p<0.001)$ e atitudes em relação à alteração com a imagem corporal $(r=$ 0.38; $p<0.001)$. A insatisfação corporal não se associou significativamente com a qualidade de vida $(p=0.524)$.

A análise comparativa dos escores de qualidade de vida em função dos níveis de IMC, unicamente evidenciou um efeito significativo no momento 2 (pós-menarca) ( $p$ $<0.05)$, tendo grupo de adolescentes insatisfeitas com sua imagem corporal revelado níveis de qualidade de vida inferiores ao grupo de adolescentes satisfeitas $(48.55 \pm 7.19$ vs. $53.09 \pm 7.92 ; \quad$ respectivamente). 
A análise longitudinal dos níveis de renda familiar e IMC indicou aumento significativo ao longo dos três momentos da menarca nomeadamente: renda familiar $(F=47.17 ; p<$ $\left.0.001 ; \eta^{2}=0.26\right)$ e IMC $(F=15.62 ; p<0.001$; $\left.\eta^{2}=0.11\right)$.

Tabela 3

Análise de correlação entre renda familiar, IMC, insatisfação da imagem corporal e qualidade de vida em momentos distintos da menarca.

Renda familiar/Dimensões da IIC/QV

IMC/Escore total da IIC

IMC/Dimensões específicas da IIC

Auto perceção da forma/Aparência corporal

Perceção comparativa da IC

Atitudes em relação à alteração com a IC IIC/QV

\begin{tabular}{ccccccc}
\multicolumn{2}{c}{ Momento 1 } & \multicolumn{2}{c}{ Momento 2 } & \multicolumn{2}{c}{ Momento 3 } \\
\hline$r$ & $p$ & $r$ & $P$ & $r$ & $P$ \\
\hline- & - & - & - & - & - \\
0.36 & $<0.001$ & 0.44 & $<0.001$ & 0.44 & $<0.001$
\end{tabular}

$0.39<0.001 \quad 0.42<0.001 \quad 0.44<0.001$

$0.24 \quad 0.005 \quad 0.26<0.001 \quad 0.32<0.001$

$\begin{array}{llllll}0.20 & 0.018 & 0.37<0.001 & 0.38<0.001\end{array}$

$0.816 \quad-\quad 0.240 \quad-\quad 0.540$

Momentos 1, 2 e 3-Momentos da menarca; $r-\bar{r}$ de Pearson; $p$-probalilidade; IIC-insatisfação da imagem corporal; QV-qualidade de vida; IMC-índice de massa corporal; IC-imagem corporal.

Relativamente à variação temporal da insatisfação corporal, a ANOVA de medidas repetidas não revelou significância para os domínios da autopercepção da forma/aparência corporal e percepção comparativa da imagem corporal, unicamente indicou um efeito significativo na subescala das atitudes em relação às alterações com a imagem corporal ( $F$ $\left.=8.50 ; p<0.001 ; \eta^{2}=0.06\right)$. A análise post hoc de Bonferroni apontou que estes níveis de insatisfação corporal diminuíram significativamente entre os momentos 1 e 2 ( $p$ $=0.008)$ e 1 e $3(p=0.002)$, não existindo diferenças significativas entre os momento $2 \mathrm{e}$ $3(p=0.736)$.

No que concerne às medidas de qualidade de vida ao longo dos três momentos, os resultados indicaram um efeito significativo ( $F$ $\left.=4.76 ; p=0.013 ; \eta^{2}=0.03\right)$. Os valores do escore de qualidade de vida aumentaram de $50.79 \pm 9.17$ no momento 1 para $52.72 \pm 7.94$ no momento $2(p=0.037)$, tendo diminuído do momento 2 para $49.96 \pm 10.32$ no momento $3(p=0.007)$. Não se observaram diferenças significativas entre os momentos $1 \mathrm{e}$ $3(p=1.00)$.

\section{DISCUSSÃO}

Os resultados indicaram uma redução do IMC e uma diminuição da insatisfação da imagem corporal, durante todo o período de acompanhamento longitudinal do estudo. Ademais, comprovou-se que o IMC relacionouse positivamente com a insatisfação corporal nos três momentos da menarca. Scherer, Martins, Pelegrini, Matheus, e Petroski (2010) corroboram com os resultados deste estudo ao verificarem que a insatisfação com a imagem corporal apresentou associação com o IMC em adolescentes. Estes autores têm fundamentado que a maioria das adolescentes e mulheres jovens se mostram insatisfeitas com a sua imagem corporal, mesmo que revelem menor excesso de peso corporal. Em um estudo transversal em populações parecidas, Santos et al. (2009) constataram aumento do nível de insatisfação da imagem corporal em adolescentes na pós-menarca, mesmo utilizando um instrumento (Stunkard, Sorenson, \& Schlusinger, 1983) para coleta de dados diferente ao usado no nosso estudo (BSQ), mas apresentando um IMC normal. Além disto, Santos et al. (2012) em concordância com os resultados do nosso estudo, utilizandose do mesmo instrumento (BSQ) para verificar 
a insatisfação da imagem corporal, encontraram adolescentes com baixa insatisfação com a imagem corporal e um IMC normal nos momentos antes e depois da menarca.

No momento 2 a comparação da qualidade de vida em função dos níveis de IMC demonstrou que o grupo de adolescentes insatisfeitas com a imagem corporal reportou níveis de qualidade de vida menores que o grupo de adolescentes satisfeitas com a imagem corporal. Este resultado é concordante com investigações sobre a qualidade de vida relacionada à saúde de adolescentes e sua associação com os índices de IMC, que apontaram uma baixa qualidade de vida em adolescentes com sobrepeso (Kunkel, Oliveira, \& Peres, 2009). Larsson, Karlsson, e Sullivan (2002) indicam em seu estudo que o sexo feminino tem revelado baixos níveis de qualidade de vida em populações com sobrepeso e obesidade. $\mathrm{O}$ maior desenvolvimento de sobrepeso em adolescentes na pós-menarca leva a maioria das adolescentes a apresentar o desejo de perder peso devido a uma maior insatisfação com sua imagem corporal (Petroski, Velho, \& de Bem, 1999) e consequentemente, uma pior qualidade de vida.

Os níveis de renda familiar e IMC em distintos momentos da menarca indicou um aumento significativo do IMC à medida que elevou a renda familiar, mesmo que a maioria das adolescentes tenham revelado IMC normal. Gomes, Anjos, e Vasconcellos (2009) corroboram com esses resultados ao relatarem uma tendência positiva de aumento proporcional de adolescentes com sobrepeso e obesidade, conforme se eleva a renda familiar. No entanto, tem-se sugerido que essa associação também pode ser encontrada entre adolescentes que pertencem a famílias de situação socioeconômica menos privilegiada (Roman et al., 2009). E ainda, outros estudos mostraram que a antecipação da menarca em adolescentes pode estar associada com a melhora do nível socioeconômico e maior prevalência de excesso de peso e obesidade, nesta fase da vida (Hernández et al., 2007; Laitinen, Power, \& Järvelin, 2001).

Outro achado importante do nosso estudo foi uma menor insatisfação da imagem corporal ao longo dos três momentos da menarca, segundo os níveis de IMC revelado pela diminuição do sobrepeso. Tais indicações deste resultado demonstram, sobremaneira, o menor nível insatisfação da imagem corporal no momento 2 quando comparado ao momento 1 da menarca. Entretanto, em relação ao resultado constatado pela diminuição significativa longitudinal da insatisfação da imagem corporal entre o momento 1 e 3 podese se afirmar que é um dos principais achados deste estudo e que colaboram para preencher uma lacuna do conhecimento científico.

Santos et al. (2012) também compararam os níveis de satisfação da imagem corporal de adolescentes em dois momentos diferentes da menarca, um antes e o outro depois da menarca. Os autores concluíram que o IMC normal predominou entre as adolescentes nos dois momentos, mesmo apresentando uma menor insatisfação com a imagem corporal depois da menarca. Slade (1994) descreve a imagem corporal como uma flutuante representação mental do tamanho, contorno e aparência do corpo que é influenciada por uma variedade de fatores históricos, culturais, sociais, individuais e biológicos que atuam variavelmente ao longo do tempo. Verifica-se que a diminuição dos níveis de insatisfação com a imagem corporal, tem sido frequentemente definida com uma diferença entre a percepção, o tamanho do corpo e a forma desejada (Bosi, Luiz, Morgado, Costa, \& Carvalho, 2006) e que ao longo do tempo e em diferentes fases da menarca podem ser resultados dos fatores decorrentes da maturação sexual destas adolescentes (Lourenço \& Queiroz, 2010).

No que se refere às alterações da qualidade de vida ao longo dos três momentos da menarca, os resultados indicaram um aumento significativo entre o momento 1 e 2 da 
menarca, tendo diminuído do momento 2 para o 3. Lee (2008), Rembeck e Gunnarsson (2004) verificaram resultados de atitudes positivas em relação ao comportamento de adolescentes na pós-menarca e justificaram tal ocorrência em virtude dos efeitos benéficos decorrentes da maturação. Rembeck e Gunnarsson (2004) advertem que as adolescentes se sentem orgulhosas ao menstruar, diminuem o nível de ansiedade em função de ter seu primeiro período menstrual e ficando felizes com a sua primeira menstruação. Lee (2008) relata experiências positivas depois da menarca e indicam menos sentimentos de vergonha e de insegurança, causada em muitas mulheres jovens, em torno do segredo do seu primeiro período menstrual. Parece que o comportamento positivo em relação à menarca reflete na melhoria da qualidade de vida.

Entretanto, um revelador achado do nosso estudo indica que no momento 2 da menarca, as adolescentes retornaram aos níveis iniciais de qualidade de vida avaliada no momento 1. Gordia, Quadros, Campos e Junior (2009) explicam este acontecimento dizendo que as adolescentes com o passar do tempo, mudam alguns valores comportamentais e adquirem uma nova e diferenciada visão crítica em relação a qualidade de vida, no que diz respeito à questões referentes a imagem corporal e auto estima. Isto posto, supõe-se a importância da menarca como uma referência para qualidade de vida das adolescentes ao longo do tempo. Possíveis implicações poderiam ser apresentadas acerca da importância prática do estudo, como a necessidade de intervenções de programas sociais na educação, na psicologia e na saúde no sentido de melhorar a imagem corporal e a qualidade de vida de adolescentes nas diferentes fases da menarca.

Este estudo apresenta possíveis limitações relacionadas à falta de informações da relação materna a respeito do momento da menarca e a perda amostral que aconteceu em função das greves dos professores e da evasão das adolescentes por diversos motivos durante os 30 meses de coleta de dados.

\section{CONCLUSÃo}

A partir dos resultados constatou-se no presente estudo, que as adolescentes revelaram uma menor insatisfação com a imagem corporal com o passar do tempo, evidenciada por um IMC normal; e ao longo do tempo a baixa insatisfação da imagem corporal revelouse pela associação positiva da renda familiar e do IMC. Já a qualidade de vida apresentou-se melhor entre o momento 1 e 2 da menarca, mas entre o momento 2 e 3 ocorreu uma diminuição da qualidade de vida das adolescentes.

Ademais, sugere-se a realização de estudos experimentais que contribuem para identificar a insatisfação com a imagem corporal de adolescentes com a menarca; estudos que enfoquem a satisfação e insatisfação em diferentes grupos populacionais relacionando a fatores como socioeconômicos, religiosos, nutricionais e comportamentais. Uma vez que este assunto deve ser mais explorado para o avanço do conhecimento relacionado a esta temática na área da saúde.

\section{Agradecimentos:}

Nada a declarar

\section{Conflito de Interesses:}

Nada a declarar.

\section{Financiamento:}

Nada a declarar.

\section{REFERÊNCIAS}

Al-Sahab, B., Ardern, C. I., Hamadeh, M. J., \& Tamim, H. (2010). Age at menarche in Canada: results from the National Longitudinal Survey of Children \& Youth. BMC Public Health, $\quad 10(1), \quad 736$. http://doi.org/10.1186/1471-2458-10-736

Assumpção, F., Kuczynski, E., Sprovieri, M. H., \& Aranha, E. M. G. (2000). Escala de avaliação de qualidade de vida: validade e confiabilidade de 
uma escala para qualidade de vida em crianças de 4 a 12 anos. Arquivos de Neuro-Psiquiatria, $58(1)$, 119-127. http://doi.org/10.1590/S0004282X2000000100018

Bosi, M. L. M., Luiz, R. R., Morgado, C. M. da C., Costa, M. L. dos S., \& Carvalho, R. J. de. (2006). Autopercepção da imagem corporal entre estudantes de nutrição: um estudo no município do Rio de Janeiro. Jornal Brasileiro de Psiquiatria, 55(2), 108-113. http://doi.org/10.1590/S004720852006000200003

Castilho, S. D., Pinheiro, C. D., Bento, C. A., BarrosFilho, A. de A., \& Cocetti, M. (2012). Tendência secular da idade da menarca avaliada em relação ao índice de massa corporal. Arquivos Brasileiros de Endocrinologia \&amp; Metabologia, 56(3), 195-200. http://doi.org/10.1590/S000427302012000300008

Cole, T. J., Bellizzi, M. C., Flegal, K. M., \& Dietz, W. H. (2000). Establishing a standard definition for child overweight and obesity worldwide: international survey. BMJ, 320(7244), 1240. http://doi.org/10.1136/bmj.320.7244.1240

Conde, W. L., \& Monteiro, C. A. (2006). Body mass index cutoff points for evaluation of nutritional status in Brazilian children and adolescents. Jornal de Pediatria, 82(4), 266-272. http://doi.org/10.2223/JPED.1502

Conti, M. A., Cordás, T. A., \& Latorre, M. do R. D. de O. (2009). A study of the validity and reliability of the Brazilian version of the Body Shape Questionnaire (BSQ) among adolescents. Revista Brasileira de Saúde Materno Infantil, 9(3), 331-338. http://doi.org/10.1590/S151938292009000300012

Cooper, P. J., Taylor, M. J., Cooper, Z., \& Fairbum, C. G. (1987). The development and validation of the body shape questionnaire. International Journal of Eating Disorders, 6(4), 485-494. http://doi.org/10.1002/1098108X(198707)6:4<485::AIDEAT2260060405 > 3.0.CO;2-O

Del Ciampo, L. A., \& Del Ciampo, I. R. L. (2010). Adolescência e imagem corporal. Adolescência \& Saúde, 7(4), 55-59.

Gomes, F. da S., Anjos, L. A. dos, \& Vasconcellos, M. T. L. de. (2009). Associação entre o estado nutricional antropométrico e a situação sócioeconômica de adolescentes em Niterói, Rio de Janeiro, Brasil. Cadernos de Saúde Pública, 25(11),

2446-2454.

http://doi.org/10.1590/S0102311X2009001100014

Gordia, A. P., Quadros, T. M. B. de, Campos, W. de, \& Vilela Júnior, G. de B. (2009). Qualidade de vida de adolescentes da rede particular de en- sino: comparação entre gêneros. Revista Brasileira de Qualidade de Vida, 1(2), 16-24. http://doi.org/10.3895/S217508582009000200003

Hernández, M. I., Unanue, N., Gaete, X., Cassorla, F., \& Codner, E. (2007). Age of menarche and its relationship with body mass index and socioeconomic status. Revista médica de Chile, 135(11), 1429-1436. http://doi.org/10.4067/S003498872007001100009

Hosny, L. A., El-Ruby, M. O., Zaki, M. E., Aglan, M. S., Zaki, M. S., Gammal, M. A. El, \& Mazen, I. M. (2005). Assessment of Pubertal Development in Egyptian Girls. Journal of Pediatric Endocrinology and Metabolism, 18(6), 577584.

http://doi.org/10.1515/JPEM.2005.18.6.577

Kunkel, N., Oliveira, W. F. de, \& Peres, M. A. (2009). Excesso de peso e qualidade de vida relacionada à saúde em adolescentes de Florianópolis, SC. Revista de Saúde Pública, 43(2), 226-235. http://doi.org/10.1590/S003489102009005000012

Laitinen, J., Power, C., \& Järvelin, M. R. (2001). Family social class, maternal body mass index, childhood body mass index, and age at menarche as predictors of adult obesity. The American Journal of Clinical Nutrition, 74(3), 287294.

Larsson, U., Karlsson, J., \& Sullivan, M. (2002). Impact of overweight and obesity on healthrelated quality of life--a Swedish population study. International Journal of Obesity and Related Metabolic Disorders, 26(3), 417-424. http://doi.org/10.1038/sj.ijo.0801919

Lee, J. (2008). Bodies at Menarche: Stories of Shame, Concealment, and Sexual Maturation. Sex Roles, 60(9-10), 615-627. http://doi.org/10.1007/s11199-008-9569-1

Lourenço, B., \& Queiroz, L. B. (2010). Crescimento e desenvolvimento puberal na adolescência. Revista de Medicina, 89(2), 70-75.

Manificat, S., \& Dazord, A. (1997). Evaluation de la qualité de vie de l'enfant : validation d'un questionnaire, premiers résultats. Neuropsychiatrie de l'enfance et de l'adolescence, 45(3), 106114.

Orden, A. B., Vericat, A., \& Apezteguía, M. C. (2011). Age at menarche in urban Argentinian girls: association with biological and socioeconomic factors. Anthropologischer Anzeiger, 68(3), 309-322. http://doi.org/10.1127/0003$5548 / 2011 / 0109$

Petroski, E. L., Velho, N. M., \& de Bem, M. F. L. (1999). Idade de menarca e satisfação com o peso corporal. Revista Brasileira Cineantropometria e Desempenho Humano, 1(1), 30-36.

Rembeck, G., \& Gunnarsson, R. (2004). Improving Pre- and Postmenarcheal 12-Year-Old Girls' 
Attitudes Toward Menstruation. Health Care for Women International, 25(7), 680-698. http://doi.org/10.1080/07399330490458033

Roman, E. P., Ribeiro, R. R., Guerra-Júnior, G., \& Barros-Filho, A. de A. (2009). Antropometria, maturação sexual e idade da menarca de acordo com o nível socioeconômico de meninas escolares de Cascavel (PR). Revista da Associação Médica Brasileira, 55(3), 317-321. http://doi.org/10.1590/S010442302009000300026

Santos, M. L. B., Monteiro, L. A. C., Ferreira, M. E. C., Sousa, M. S. C., Damasceno, V. O., Miranda, H., \& Novaes, J. S. (2012). Níveis de satisfação da imagem corporal de adolescentes antes e depois da menarca. Revista Terapia Manual, 10(49), 239-245.

Santos, M. L. B., Monteiro, L. A. C., Silva, M. F., Sousa, M. do S. C. de, \& Novaes, J. da S. (2009). Imagem corporal e níveis de insatisfação em adolescentes na pós-menarca. Revista Da Educação Física/UEM, 20(3), 333-341. http://doi.org/10.4025/reveducfisv20n3p333341
Scherer, F. C., Martins, C. R., Pelegrini, A., Matheus, S. C., \& Petroski, E. L. (2010). Imagem corporal em adolescentes: associação com a maturação sexual e sintomas de transtornos alimentares. Jornal Brasileiro de Psiquiatria, 59(3), 198-202. http://doi.org/10.1590/S004720852010000300005

Slade, P. D. (1994). What is body image? Behaviour Research and Therapy, 32(5), 497-502. http://doi.org/10.1016/0005-7967(94)901368

Sloboda, D. M., Hart, R., Doherty, D. A., Pennell, C. E., \& Hickey, M. (2007). Age at menarche: Influences of prenatal and postnatal growth. The Journal of Clinical Endocrinology and Metabolism, 92(1), 46-50. http://doi.org/10.1210/jc.2006-1378

Stunkard, A. J., Sorenson, T., \& Schlusinger, F. (1983). Use of the Danish adoption register for the study of obesity and thinness. Em S. S. Kety (Ed.), Genetics of Neurological and Psychiatric Disorders (pp. 115-120). New York: Raven Press.

Todo o conteúdo da revista Motricidade está licenciado sob a Creative Commons, exceto quando especificado em contrário e nos conteúdos retirados de outras fontes bibliográficas. 